\title{
Source placement error for permanent implant of the prostate
}

\author{
Peter L. Roberson \\ University of Michigan, Ann Arbor, Michigan 48109 and Providence Hospital, Southfield, Michigan 48075 \\ Vrinda Narayana \\ Providence Hospital, Southfield, Michigan 48075 \\ Daniel L. McShan \\ University of Michigan, Ann Arbor, Michigan 48109 \\ Raymond J. Winfield \\ Providence Hospital, Southfield, Michigan 48075 \\ P. William McLaughlin \\ University of Michigan, Ann Arbor, Michigan 48109 and Providence Hospital, Southfield, Michigan 48075
}

(Received 8 March 1996; accepted for publication 31 October 1996)

The performance of ultrasound (US) and fluoroscopic-guided permanent ${ }^{125}$ I source implant of the prostate using CT identification of the source positions has been evaluated. Marker seeds were implanted during the planning study to assist in the alignment of the US and CT prostate volumes for treatment planning and to guide the placement of needles. The relative positions of the needles and marker seeds were checked by fluoroscopy. A postimplant CT study was used to input the radioactive source positions and to register the sources relative to the preimplant CT and US prostate volumes and the planned source distribution. Source placement errors observed were categorized as: (1) source-to-source spacing differences; (2) needle placement error, both depth and position; and (3) seed splaying, particularly near the prostate periphery. Errors due to source splaying and spacing were in part attributed to prostate motion. Later refinements included fixedspaced string sources, for which placement errors were smaller than for unattached sources. However, source placement errors due to needle placement error and prostate motion remained unchanged. (C) 1997 American Association of Physicists in Medicine. [S0094-2405(97)02402-4]

Key words: prostate implant, ultrasound, CT, dosimetry, 3D treatment planning

\section{INTRODUCTION}

Permanent implants of the prostate using ${ }^{125} \mathrm{I}$ or ${ }^{103} \mathrm{Pd}$ are being performed in many centers. ${ }^{1}$ Ultrasound (US) and fluoroscopic-guided needle placement has improved the reliability of the source placement by improving the visualization of the prostate relative to the needle placement during the procedure. ${ }^{2}$ The quality of the implant depends on the dosimetric evaluation; the dose delivered to the prostate compared to the dose delivered to the normal tissues. To perform postimplant dosimetric evaluations, information on source placement relative to the prostate and normal tissues was obtained. This information was also used to perform an evaluation of the errors in source placement, with the goal of minimizing source placement error and ultimately maximizing the dose to the target organ compared to neighboring normal tissues.

In optimizing the dose distribution, many treatment plans may be considered containing variations in source density and/or source strength. A treatment plan may be designed to optimize the target dose distribution. However, the actual dose distribution would vary considerably from the ideal if the source placement was not perfect. An evaluation of typical source placement errors may aid in the comparison of optimized treatment plans by determining potential risks of poor results due to source placement error.
We have performed postimplant dosimetric evaluations of US and fluoroscopic-guided permanent implants of the prostate using CT identification of the source positions. We have measured and analyzed the contributors to the source placement error so that expected systematic and random errors may be included into the treatment planning process.

\section{METHODS}

Anatomical data were collected during a planning study performed in the operating room under spinal anesthesia. Serial US images (Fig. 1) with prostate contours were obtained. For early patients, approximately seven marker (nonradioactive) seeds were implanted, using three each in two needles inserted to the depth of the prostate base and one to the depth of the prostate apex (Fig. 2). The three marker seeds per needle were used to define the implant axis in addition to the depth of the base. The AP view relative to the marker seeds was defined perpendicular to the implant axis. Patients treated later received six marker seeds in three positions, two at the base and one at the apex. Two markers per position were used to better visualize their location under US. Template holes approximately $1 \mathrm{~cm}$ medial to the prostate edges were chosen for the marker seed implantation to minimize seed placement artifact caused by prostate motion. 


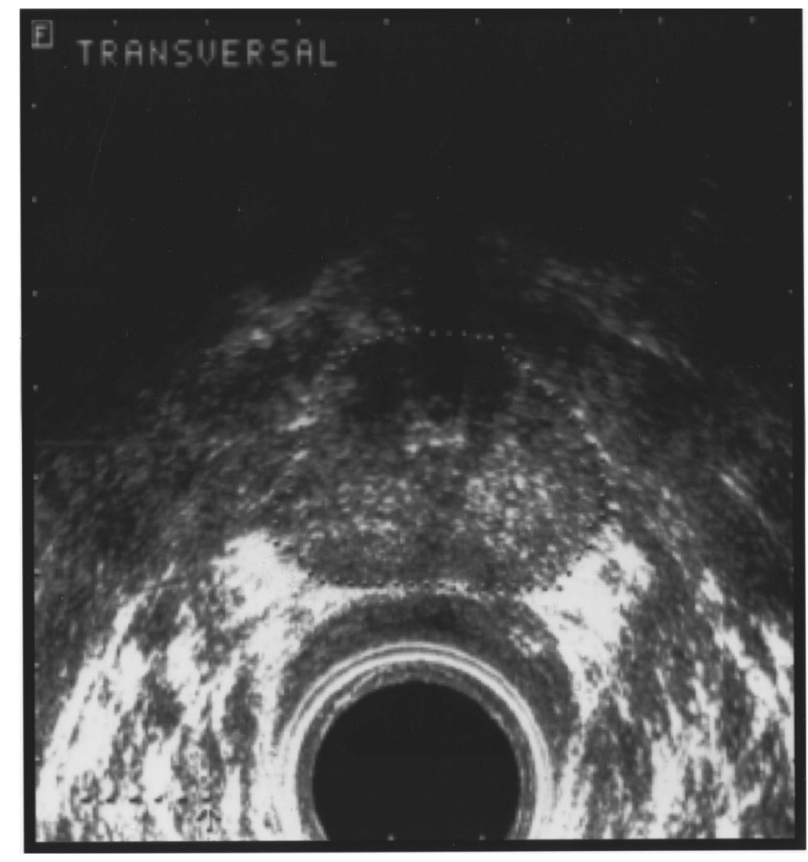

FIG. 1. US Image. An axial slice showing the contoured prostate structure. Sections were taken at $0.5 \mathrm{~cm}$ intervals.

A preimplant CT was obtained using $3 \mathrm{~mm}$ cut spacings to localize the marker seeds relative to the prostate. The CT prostate volume was registered relative to the US volume using University of Michigan treatment planning software (UMPLAN), ${ }^{3}$ aligning the prostate base and posterior surface (the two most reliable surfaces) (Fig. 3). The alignment was accomplished using interactive graphics and contour comparison on orthogonal planes. The US/CT registration was adjusted, when necessary, to account for the relative alignment of the marker seeds with the template hole positions

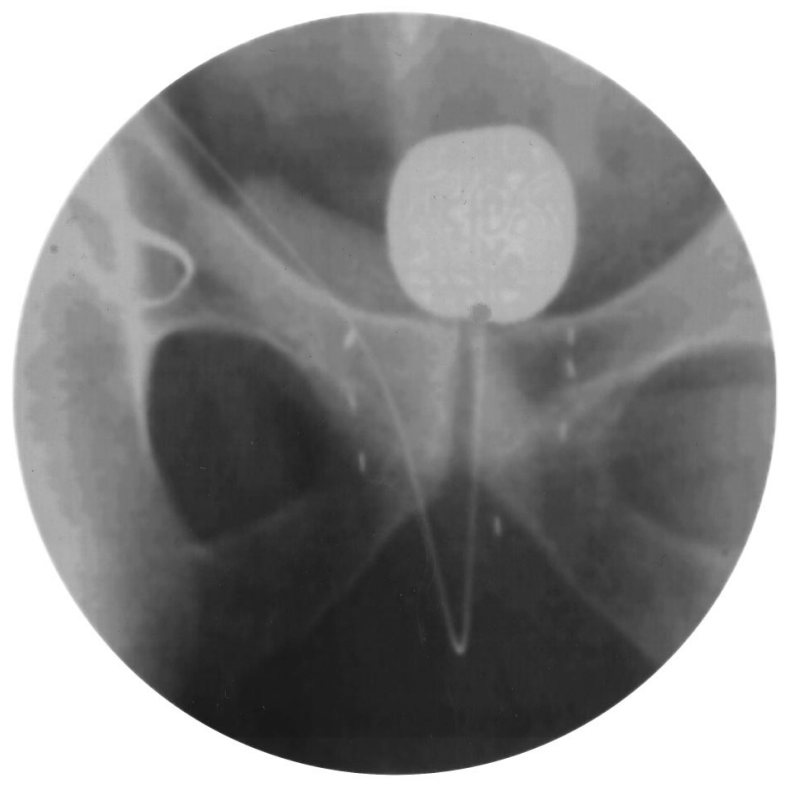

FIG. 2. Fluoroscopic view of marker seed distribution. Marker seeds were placed during the planning study.
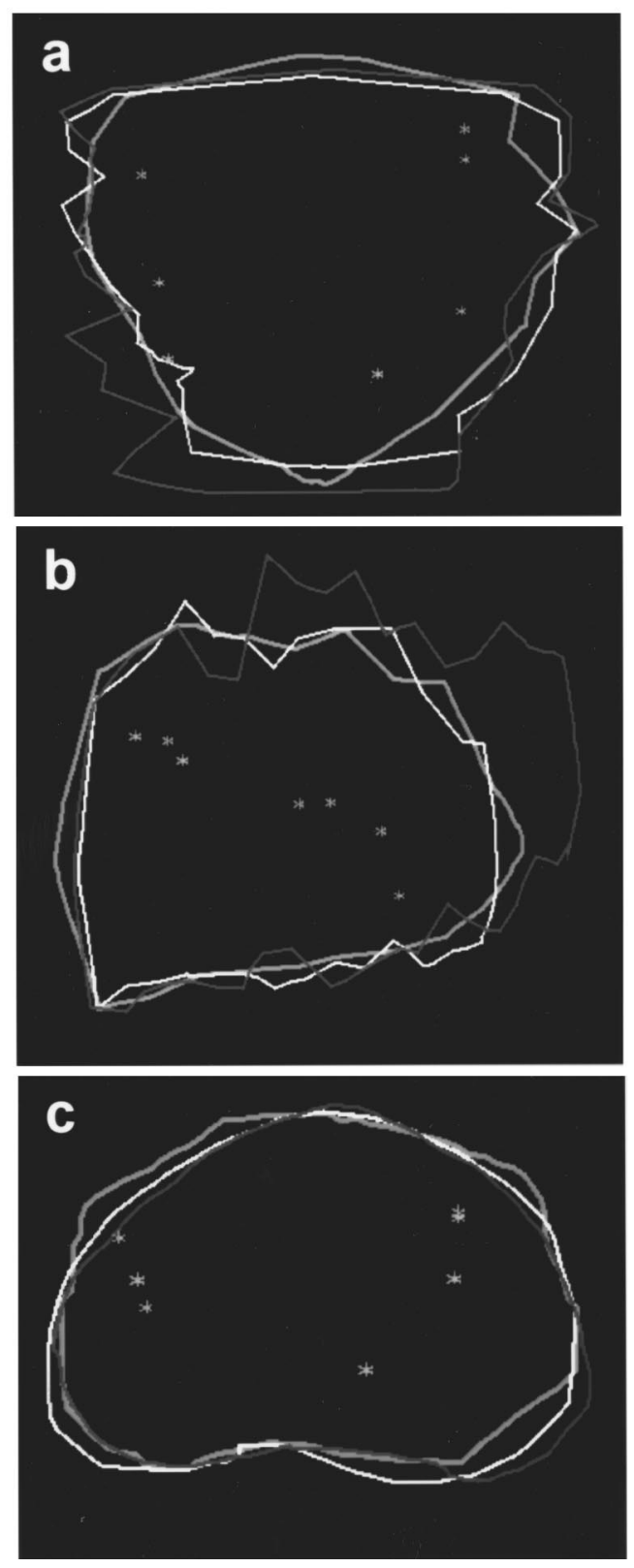

FIG. 3. Marker seed locations. Marker seeds relative to the US (thick line), preimplant CT (thin, dark line), and postimplant CT (thin, light line) volumes on (a) coronal, (b) sagittal, and (c) axial views. Views of the marker seeds relative to the prostate volumes were used to check needle placement.

and the axis of the seeds relative to the US axial data set. The relationship between the marker seeds and volumes was used during the implant procedure to help adjust needle depth placement.

The US and CT prostate volumes were used for treatment planning (Fig. 4). Initial plans used a uniform $1 \mathrm{~cm}$ spacing of the seed distribution and uniform activity. Later plans used a smaller number or lighter activity seeds in the center. The goal was to encompass the prostate volume with the prescribed isodose surface without excessive dose deposited centrally. The US prostate changed with probe position. Some contours contained eccentric, asymmetric areas that were not reproduced at time of implant. The dose prescrip- 

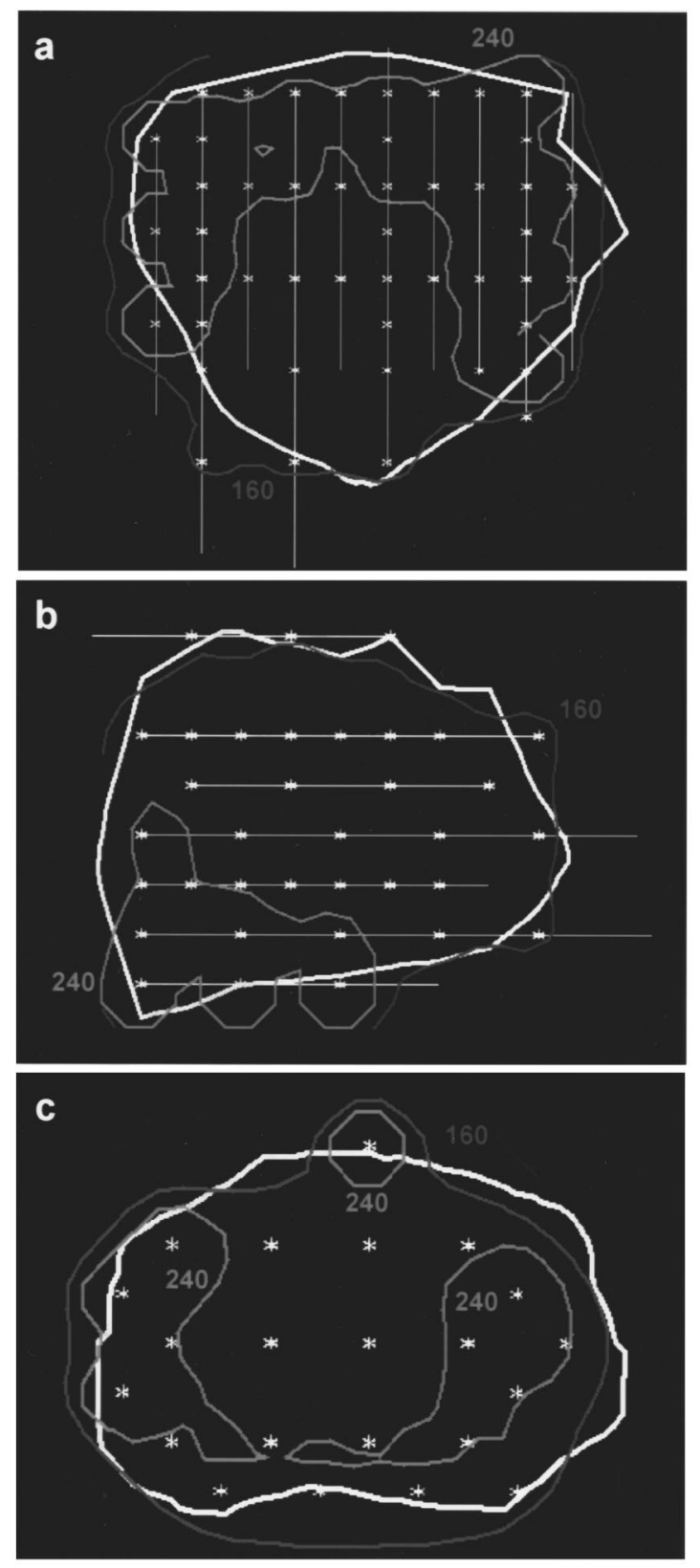

FIG. 4. Implant plan. Isodose lines for 160 and $240 \mathrm{~Gy}$ are shown superimposed on the (a) coronal, (b) sagittal, and (c) axial planes displaying the US prostate contours.

tion surface was not designed to encompass eccentric areas, as long as the prescription coverage exceeded $95 \%$ of the US prostate volume. The implanted activity was increased by $15 \%$ to help account for the effects of source placement error.

The US image positions were reproduced prior to needle insertion. Sources were loaded at time of implant using disposable needles. Both individual sources and fixed-space source strings (RAPID Strand, Medi-Physics, Inc., Arlington Heights, IL) were used. Initially, individual sources were loaded using a minimal amount of wax at the needle tip. Anusol HC was used with string sources, as recommended by the manufacturer, and was later used with individual

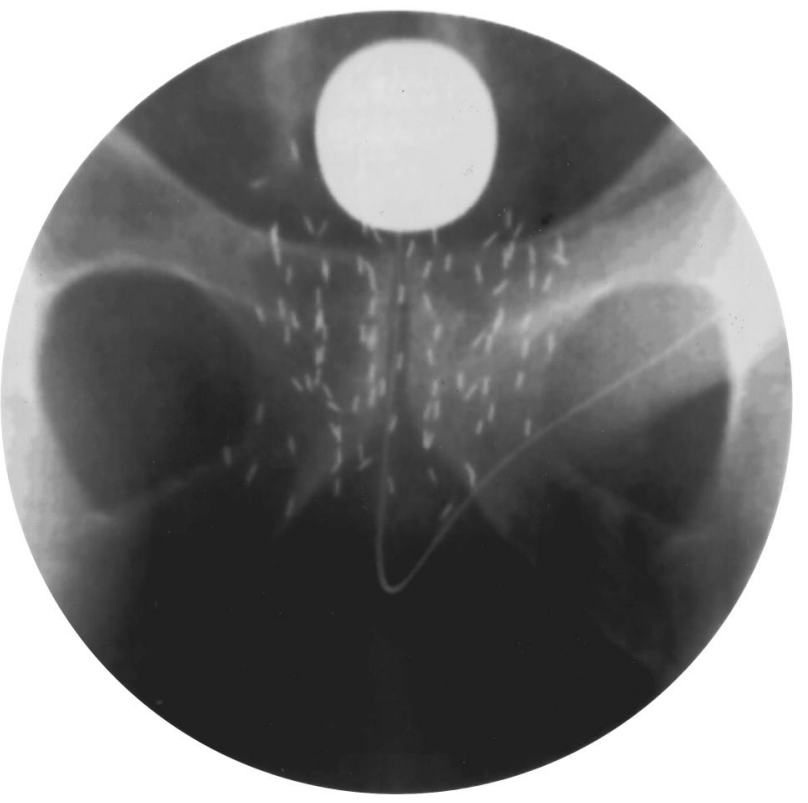

FIG. 5. Fluoroscopic view of final source distribution.

sources yielding improved results. The needle position was determined using the template hole and flash on the US screen indicating needle alignment and depth. The needle depth was checked and adjusted using fluoroscopy and longitudinal US view, checking the relative position of the needle and marker seeds by fluoroscopy. Errors due to prostate longitudinal and rotational motions were partially compensated by needle placement technique. ${ }^{4}$ The effect of longitudinal prostate motion was reduced by pushing the needle through the prostatic tissue and pulling it back to the proper position. The effect of prostate rotation was reduced by placing the US flash image for peripheral needles medial with respect to the planned position. Stabilizing needles were used provided they did not excessively distort the prostate anatomy. Each needle insertion was checked for proper source deposition. Extra seeds were implanted if an abnormal drop-off was noted. An x-ray image of the final source distribution was taken (Fig. 5).

For cases planned with improved target dose uniformity, larger activity sources or greater numbers of sources per unit volume were placed on the prostate periphery. The quality of the implant was more dependent on the peripheral sources, which were also more difficult to position precisely. Individual nonapical sources implanted outside of the prostate capsule were prone to movement and were not considered reliable. An effort was made to ensure that all sources were implanted within the capsule. This was less of a problem when using string sources since a source outside the capsule was typically tethered to sources inside the capsule.

A postimplant CT data set was obtained up to four weeks following the implant using a $3 \mathrm{~mm}$ cut spacing. The postimplant prostate volume was registered with the US and preimplant volumes (Fig. 3). The US and CT prostate volumes were generated from axial contours. The volumes were aligned by superimposing the prostate surfaces at the base 

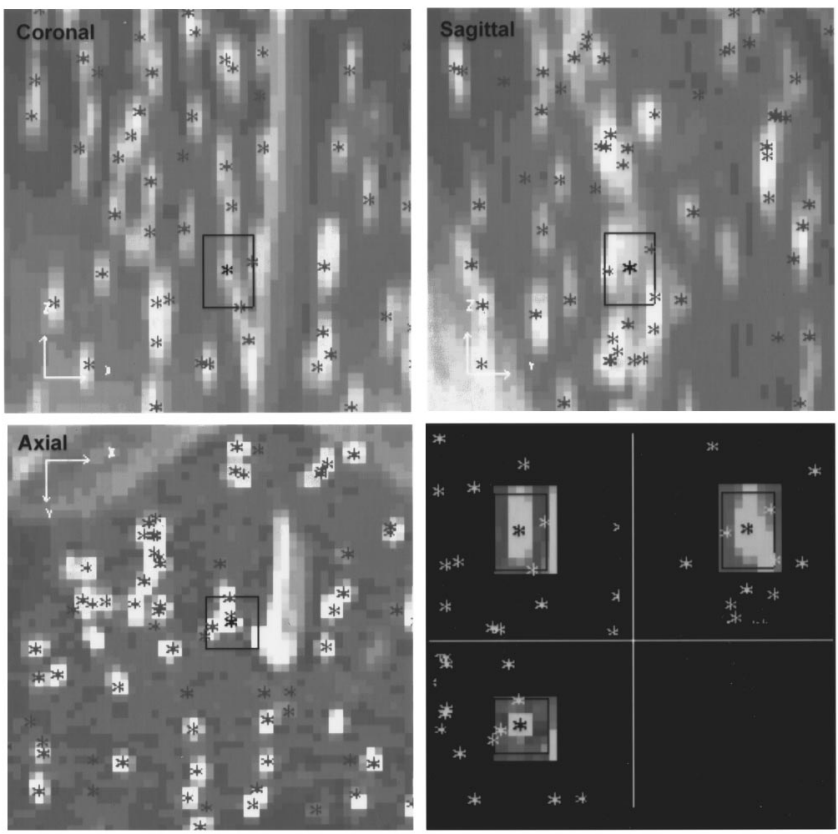

FIG. 6. Source localization from CT data set. Digitally reconstructed coronal, sagittal, and axial views were generated by collapsing a cube of CT data. The localization box was set slightly larger than the source size. The cube of CT data was reduced to the size of the localization box to confirm the location of a source (lower right panel). CT slices were taken at $3 \mathrm{~mm}$ increments. Identified source positions superimposed on orthogonal digitally reconstructed views are shown.

and adjacent to the rectum. The anterior and apical surfaces for the CT data set were less reliable due to the poor contrast between prostate and surrounding tissues on CT. The alignment was performed interactively by observing axial, sagittal and coronal contours as the US volume was adjusted. The postimplant source distribution was entered directly from the CT data set using UMPLAN software. Orthogonal views of a block of CT data were calculated, similar to digitally reconstructed radiographs (DRR) (Fig. 6). A suspected seed location was identified by placing the cursor on two of the views. The box appears automatically on the third. The source location was confirmed by shrinking the block DRR to a 5 $\mathrm{mm} \times 5 \mathrm{~mm} \times 7 \mathrm{~mm}$ volume, just large enough to confirm a solitary source (Fig. 6). If the source location was true, it was displayed within the smaller cube. Some misidentification can occur for seeds separated by less than $2 \mathrm{~mm}$. Source doublets (typically $<5 \%$ of the sources) too close for direct identification were identified from the AP view of the distribution (Fig. 5), where essentially all seeds can be resolved. Doublets typically appeared larger than a single source on the CT DRRs, so noting a doublet on the AP radiograph was enough to confirm its location. Sources were entered by moving from source to source, for each shifting to subview to confirm its location and identifying coordinates with two mouse clicks.

The registration of the postimplant $\mathrm{CT}$ volume and the seed distribution was nearly perfect, since both were entered from the same data set. The actual source distribution was rotated to visualize the needle paths (probe's eye view, Fig.

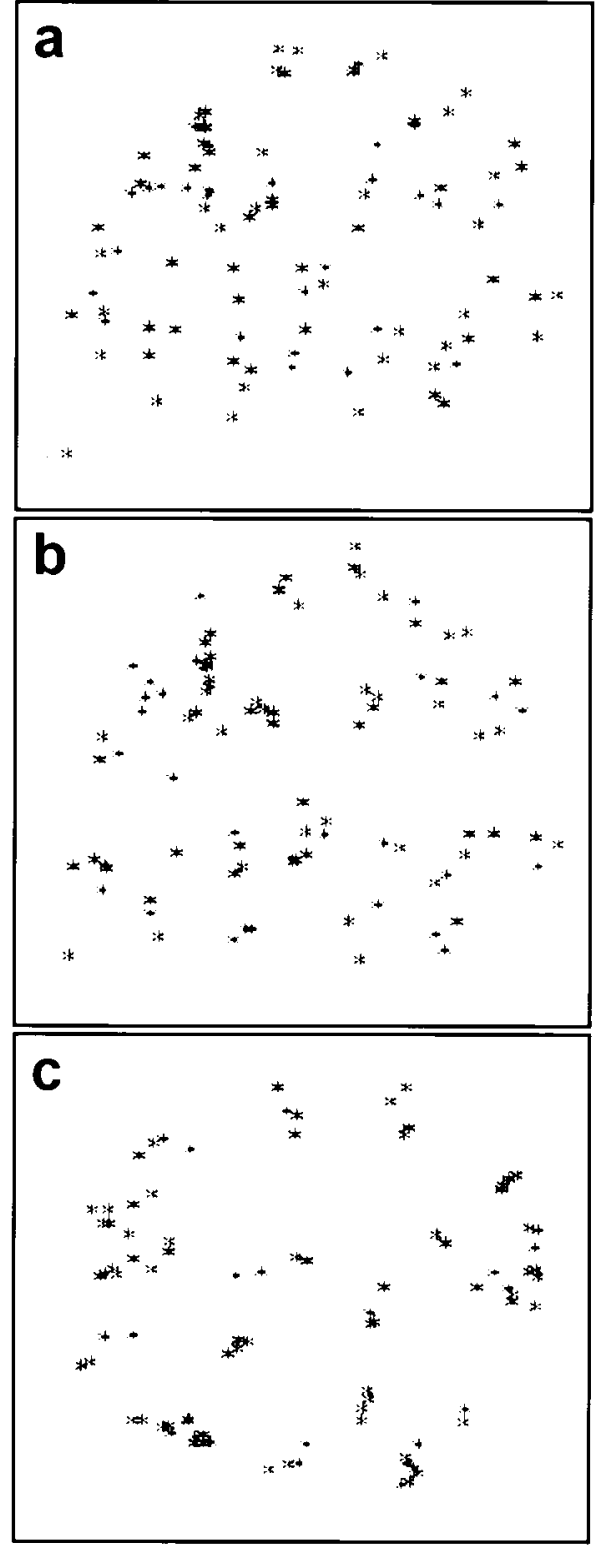

FIG. 7. Probe's eye view. Views rotated to align the axes of the needle paths to the view axis. (a) and (b) represent small relative rotations for case \#2 (individual sources), preferentially aligning anterior sources or posterior sources. The difference is primarily due to splaying. (c) Probe's eye view for case \#1 (individual sources).

7). Not all sources were uniquely identified as belonging to a particular needle path, but many needle paths were uniquely identified. The distribution of identifiable sources was compared to the planned distribution.

Errors in source placement were attributed to prostate motion (rotation and displacement) in addition to needle placement uncertainty. Errors were categorized as follows: (1) Source spacing error: sources not spaced at the planned (1 $\mathrm{cm}$ ) interval; (2) needle placement error: all sources from a needle displaced relative to the planned position; (3) splaying: sources not parallel to the planned needle axis. The source spacing errors within a needle were separated into a total length error and the absolute value of the individual 

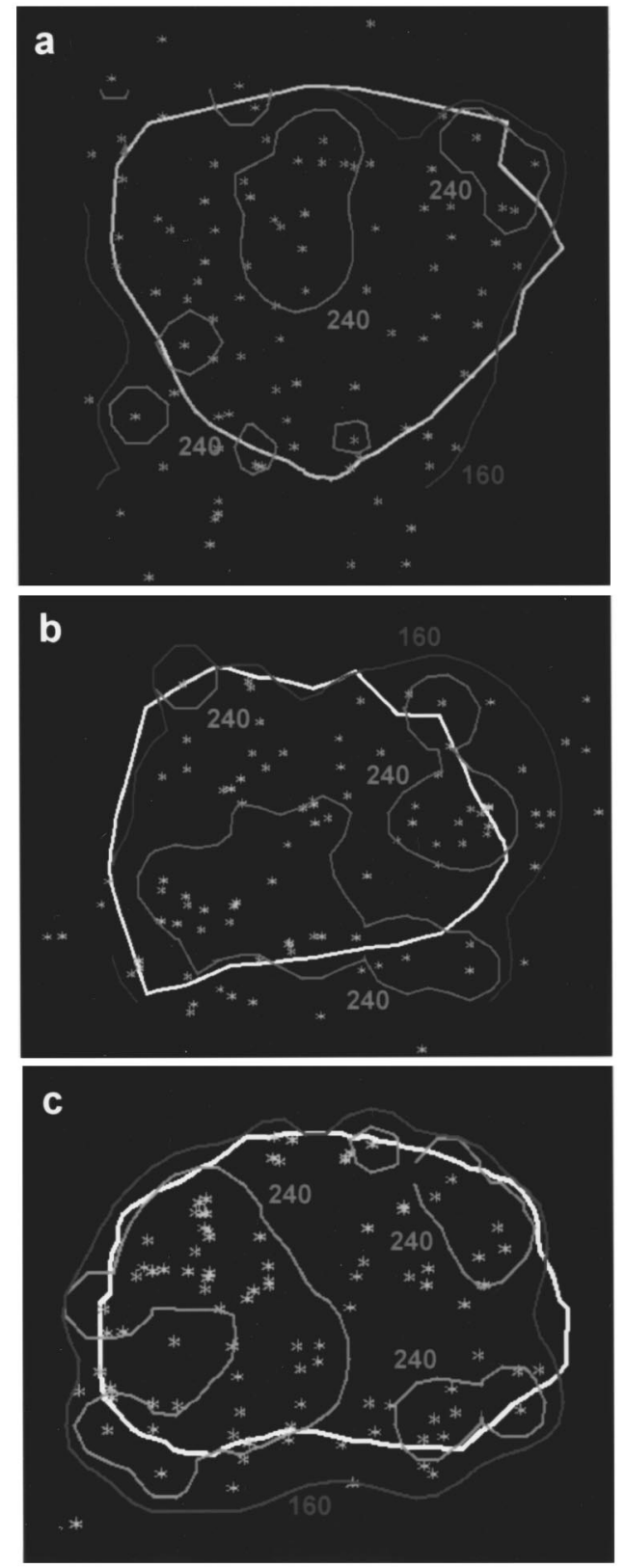

FIG. 8. Postimplant dose distributions. Isodose lines are shown for 160 and 240 Gy superimposed on the (a) coronal, (b) sagittal, and (c) axial planes for the US prostate volume.

source-to-source error. This procedure included source spacing errors even if they were both positive and negative within a needle, producing a smaller needle length error. Needle placement error was resolved into displacement (axial plane) and depth (needle axis) errors.

The source distribution was used to calculate the dose distribution and dose-volume histograms (DVH). Each source was represented by an anisotropic dose distribution ${ }^{5}$ scaled by the source activity. Each source location was identified by a single point. The source axis for anisotropy calculations was assumed to be aligned with the needle path. The air-kerma rate constant was $0.036 \mu \mathrm{Gy} \mathrm{m}^{2} \mathrm{MBq}^{-1} \mathrm{~h}^{-1}$.

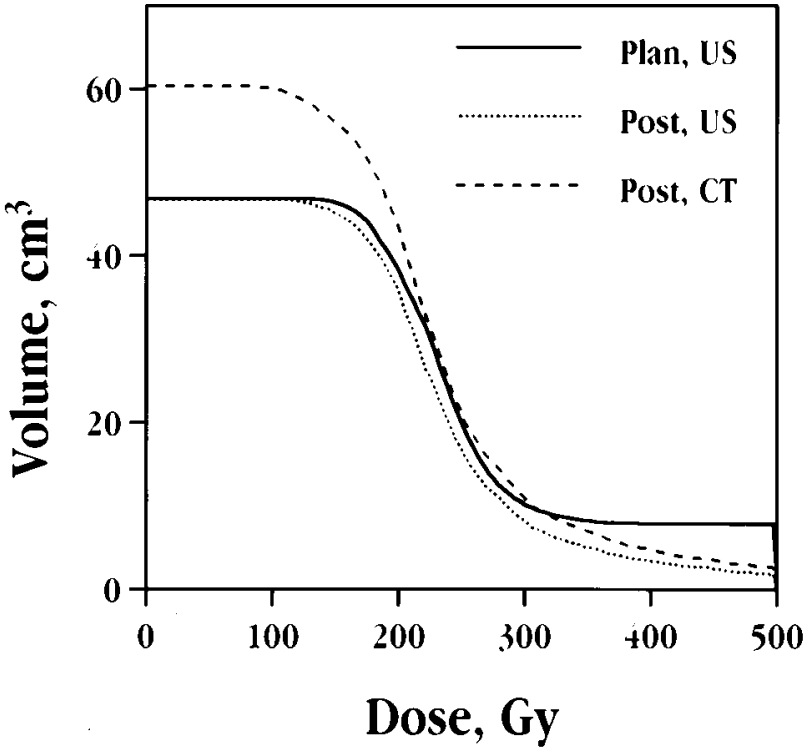

FIG. 9. Dose-volume histograms. Dose-volume histograms are shown for the planned and actual source distributions using the preimplant US volume and for the actual source distribution using the post implant CT volume. The postimplant CT volume was typically larger than the US volume.

\section{RESULTS}

Calculated dose distributions for orthogonal views are presented in Fig. 8. Dose-volume histograms for USplanned source distributions are compared to those for the actual source distribution using the US or postimplant CT volumes in Fig. 9. The CT volumes were nearly always larger than the US volumes, particularly at the prostate apex. This was attributed to the lack of density discrimination on CT. The postimplant DVH indicates the lower doses received by the additional volume. The DVH for the actual source distribution was reasonably close to that for the planned source distribution.

Tables I and II present measured source placement errors for sources identified as belonging to a particular needle path for one case using individual sources and one using source strings, respectively. Approximately 40\% (individual sources from three cases) and 74\% (string sources from four cases) of the total number of sources implanted were identified and

TABLE I. Individual source placement error, Case \#1.

\begin{tabular}{ccccccc}
\hline \hline & \multirow{2}{*}{$\begin{array}{c}\text { Number } \\
\text { of }\end{array}$} & \multicolumn{5}{c}{ Error $(\mathrm{cm})$} \\
\cline { 3 - 7 } Needle & \begin{tabular}{c} 
sources \\
\cline { 3 - 7 }
\end{tabular} & Length & Spacing & Depth & Displacement & Splaying \\
\hline $\mathrm{A}$ & 4 & -0.4 & 0.13 & -0.4 & 0.20 & 0.55 \\
$\mathrm{~B}$ & 4 & -0.8 & 0.27 & -0.7 & 0.45 & 0.52 \\
$\mathrm{C}$ & 4 & -0.5 & 0.37 & -0.4 & 0.55 & 0.44 \\
$\mathrm{D}^{\mathrm{a}}$ & 4 & -1.8 & 0.60 & 0.4 & 0.55 & 0.77 \\
$\mathrm{E}$ & 4 & +0.2 & 0.07 & 0.3 & 0.25 & 0.43 \\
$\mathrm{~F}^{\mathrm{b}}$ & 4 & +1.4 & 0.80 & 0 & 0.80 & 0.66 \\
$\mathrm{G}$ & 4 & +0.3 & 0.10 & 0.2 & 0.55 & 0.25 \\
$\mathrm{H}$ & 4 & +0.1 & 0.03 & -0.4 & 0.60 & 0.34 \\
\hline \hline
\end{tabular}

${ }^{\mathrm{a}}$ Compressed.

${ }^{\mathrm{b}}$ Last seed dragged. 
TABLE II. String source placement error, Case \#4.

\begin{tabular}{lcccccc}
\hline \hline & $\begin{array}{c}\text { Number } \\
\text { of } \\
\text { Needle }\end{array}$ & Spacing & Depth & Displacement & Splaying \\
\cline { 3 - 7 } & sources & Length & Srror $(\mathrm{cm})$ \\
\hline $\mathrm{A}$ & 4 & -1.0 & 0.33 & -0.3 & 0.5 & 0.3 \\
$\mathrm{~B}$ & 4 & -0.3 & 0.23 & -0.8 & 0.9 & 0.1 \\
$\mathrm{C}$ & 4 & -0.6 & 0.20 & -0.4 & 0.4 & 0.4 \\
$\mathrm{D}$ & 4 & 0.1 & 0.03 & -0.7 & 0.5 & 0.7 \\
$\mathrm{E}$ & 4 & -0.9 & 0.30 & -1.1 & 0.3 & 0.3 \\
$\mathrm{~F}$ & 4 & -0.4 & 0.13 & 0.1 & 0.6 & 0.3 \\
$\mathrm{G}$ & 4 & 0.0 & 0.07 & 0.0 & 0.2 & 0.1 \\
$\mathrm{H}$ & 4 & -0.4 & 0.20 & 0.3 & 0.3 & 0.3 \\
$\mathrm{I}$ & 4 & 0.0 & 0.07 & 1.2 & 0.4 & 0.3 \\
$\mathrm{~J}$ & 3 & 0.0 & 0.30 & -0.2 & 0.7 & 0.3 \\
$\mathrm{~K}$ & 3 & -0.2 & 0.10 & 0.3 & 0.4 & 0.2 \\
$\mathrm{~L}$ & 3 & -0.1 & 0.05 & 1.3 & 0.4 & 0.2 \\
$\mathrm{M}$ & 3 & -0.5 & 0.25 & 0.6 & 0.8 & 0.2 \\
$\mathrm{~N}$ & 3 & -0.4 & 0.2 & 1.3 & 0.8 & 0.1 \\
$\mathrm{O}$ & 3 & -0.1 & 0.05 & 1.2 & 0.6 & \\
\hline \hline
\end{tabular}

${ }^{a}$ RAPID Strand, Medi-Physics, Inc., Arlington Heights, IL.

evaluated. Source spacing error was split into the discrepancy in distance from the first to the last source (length) and the discrepancy in individual source spacing. Needle placement error was represented by (1) a measurement of the difference between the nominal US drop plane (usually the base plane) and the depth of the first seed for each needle (depth); and (2) the displacement of the average source positions from the planned positions on the probe's eye view display. The source splaying distance was specified as the distance between the farthest separated seeds using the probe's eye view. This was almost uniformly also the distance from the first to the last seed in the needle. Splaying errors were found to be more severe for peripheral needles.

The average and standard deviation of individual source errors are given in Table III. Differences between cases were relatively small. Case \#3 had a shorter average string length and lower splaying. Average errors for the three cases are given on the last line. The average source distribution length was $2 \mathrm{~mm}$ different from ideal, with a considerable standard deviation $(8 \mathrm{~mm})$. This was affected by the occasional phenomenon of source distribution compression and final seed dragging, as indicated in the footnotes to Table I. Source distribution compression is believed to be due to the needle dragging the prostate tissue back as the seeds are dropped off. Final source dragging occurred when the needle dragged the last source through the prostate beyond the intended drop point. It may have been related to the amount of wax used on the needle tip, and occurred less frequently and to a lesser degree after the switch to Anusol HC.

The averages and standard deviations for string source placement errors are presented in Table IV. Results are consistent from case to case. The standard deviation from the length and the average and standard deviation for the spacing were less than for individual sources (compare to Table III). Values for the other quantities are similar.

The errors of lateral displacement and splaying are related. These are attributed to prostate motion during needle placement and removal. Since the lateral displacement error was determined from the average source position, it includes some of the effect of splaying. These errors are significant, causing the average placement error to range from $6 \mathrm{~mm}$ for the first source dropped to $2 \mathrm{~mm}$ for the last source dropped.

\section{DISCUSSION}

Source placement precision is the limiting factor for the improvement of dose distributions for permanent prostate implants. Given the current state of technology, it is not possible to implement an idealized treatment plan with sufficient precision to guarantee adequate results. The use of a $15 \%$

TABLE III. Individual source placement errors, average and standard deviation.

\begin{tabular}{|c|c|c|c|c|c|c|c|c|c|c|}
\hline \multirow[b]{3}{*}{ Case } & \multicolumn{10}{|c|}{ Error $(\mathrm{cm})$} \\
\hline & \multicolumn{2}{|c|}{ Length } & \multicolumn{2}{|c|}{ Spacing } & \multicolumn{2}{|c|}{ Depth } & \multicolumn{2}{|c|}{ Displacement } & \multicolumn{2}{|c|}{ Splaying } \\
\hline & Ave & SD & Ave & SD & Ave & SD & Ave & SD & Ave & SD \\
\hline 1 & -0.17 & 0.88 & 0.29 & 0.26 & -0.09 & 0.39 & 0.53 & 0.22 & 0.50 & 0.16 \\
\hline 2 & 0.07 & 1.1 & 0.29 & 0.15 & 0.06 & 0.69 & 0.46 & 0.20 & 0.41 & 0.17 \\
\hline 3 & -0.60 & 0.50 & 0.32 & 0.23 & -0.06 & 0.46 & 0.38 & 0.12 & 0.27 & 0.14 \\
\hline Mean & -0.23 & 0.83 & 0.30 & 0.21 & 0.02 & 0.51 & 0.46 & 0.18 & 0.39 & 0.16 \\
\hline
\end{tabular}


TABLE IV. String source placement errors, average and standard deviation.

\begin{tabular}{|c|c|c|c|c|c|c|c|c|c|c|}
\hline \multirow[b]{3}{*}{ Case } & \multicolumn{10}{|c|}{ Error $(\mathrm{cm})$} \\
\hline & \multicolumn{2}{|c|}{ Length } & \multicolumn{2}{|c|}{ Spacing } & \multicolumn{2}{|c|}{ Depth } & \multicolumn{2}{|c|}{ Displacement } & \multicolumn{2}{|c|}{ Splaying } \\
\hline & Ave & SD & Ave & SD & Ave & SD & Ave & SD & Ave & SD \\
\hline 1 & -0.32 & 0.33 & 0.17 & 0.10 & 0.17 & 0.76 & 0.55 & 0.24 & 0.26 & 0.15 \\
\hline 2 & -0.20 & 0.27 & 0.20 & 0.14 & 0.13 & 0.37 & 0.32 & 0.13 & 0.35 & 0.14 \\
\hline 3 & -0.23 & 0.35 & 0.17 & 0.10 & -0.33 & 0.60 & 0.71 & 0.33 & 0.50 & 0.61 \\
\hline 4 & -0.13 & 0.26 & 0.15 & 0.06 & -0.20 & 0.44 & 0.31 & 0.21 & 0.50 & 0.41 \\
\hline Mean & -0.22 & 0.30 & 0.17 & 0.10 & -0.06 & 0.54 & 0.47 & 0.23 & 0.40 & 0.32 \\
\hline
\end{tabular}

uniform increase in source activity to counteract the effects of source position error has been evaluated and is approximately adequate for a planned uniform source spacing with individual sources. Source spacings designed to sculpt a more uniform dose to the target volume are more susceptible to source placement error.

The measurement of source error suffered from the limited number of sources that could be unambiguously identified. The string sources were less difficult to identify because their spacing was more regular. Since not all sources were used for the error calculation, the average error could be underestimated. However, places with sources noted as misplaced during the procedure, were supplemented with additional sources at the time. This process both corrected for the worst errors and eliminated those sources from the analysis. Another reason for ambiguous source identification was two adjacent strings with errors placing them too close in proximity, which may not have indicated exceptional error. We believe that the errors as presented are representative of the average errors occurring.

The use of source string technology decreased the net placement error and/or its standard deviation in two categories, the total length of the string and spacing between string sources. Errors associated with needle placement and prostate motion (depth, displacement and splaying) remained approximately unchanged. This result is not unexpected, but it emphasizes the importance of immobilizing the prostate, or compensating for its motion.

If the string sources differed from nominal spacing, the spacings were too short. The leading cause was the spacing for the final (apical) source. This was attributed to drag pressure on the source string. As the needle was retracted, the drag of the needle within the prostate tissue tended to bring the tissue along with the needle, causing pressure countered by the rigidity of the source string. The final source in the string was in the weakest position because it sustained the greatest pressure for the longest time. This effect was the likely reason longer strings more frequently jammed in the needle during the drop-off procedure. We no longer use strings longer than five sources for this reason.

An additional component of uncertainty was due to prostate swelling secondary to trauma generated by the implant. Preliminary measurements indicate that prostate swelling can be significant, but only partially resolves over time. Swelling probably contributed to the observed source placement errors by expanding the source distribution along with the prostate, while decreasing the total dose received. Results concerning the dosimetric impact of prostate swelling appear elsewhere. ${ }^{6}$

The measurement and reduction of source placement error is the most important goal of improved treatment delivery. It is not possible to evaluate competing treatment plans for optimized dose uniformity without knowledge of potential source placement errors. However, with current placement precision, some dose distribution improvement is possible. Knowledge of placement error will allow the cautious improvement of dose distributions through differential activity loading.

Some placement errors continue to be reduced through increased experience and technique improvement. The precision of placement continues to be patient dependent, probably due to differences in prostate motion between patients. Source spacing errors have been reduced through the use of strings, but also for individual sources to a lesser extent through the use of Anusol HC instead of wax. Needle placement errors depend primarily on prostate motion. Aiming medially for peripheral needles reduces splaying. Improved use of stabilization needles may be important to limit prostate motion.

\section{ACKNOWLEDGMENTS}

The authors acknowledge the help of Amy Verhelst, BFA, for the preparation of the graphics.

\footnotetext{
${ }^{1}$ A. T. Porter, J. C. Blasko, P. D. Grimm, S. M. Reddy, and H. Ragde, "Brachytherapy for prostate cancer,', Ca. Cancer J. Clin. 45, 165-178 (1995).

${ }^{2}$ K. W. Kaye, D. J. Olson, D. J. Lightner, and J. T. Payne, "Improved technique for prostate seed implantation: Combined ultrasound and fluoroscopic guidance,', J. Endourology 6, 61-66 (1992).

${ }^{3}$ B. A. Fraass and D. L. McShan, "3-D treatment planning: I. Overview of a clinical planning system," in The Use of Computers in Radiation Therapy, edited by Bruinvis IAD (Elsevier Science, Amsterdam, 1987), pp. 273-276.

${ }^{4}$ J. C. Blasko, P. D. Grimm, and H. Ragde, "Brachytherapy and organ preservation in the management of carcinoma of the prostate," Sem. Rad. Onc. 3, 240-249 (1993).

${ }^{5}$ G. H. Hartmann, W. Schlegel, and H. Scharfenberg, "The threedimensional dose distribution of ${ }^{125}$ I seeds in tissue,' Phys. Med. Biol. 28, 693-699 (1983).

${ }^{6}$ V. Narayana, P. L. Roberson, T. Pu, H. Sandler, R. H. Winfield, and P. W. McLaughlin, "Impact of differences in ultrasound and CT volumes on treatment planning of permanent prostate implants,' Int. J. Rad. Onc. Biol. Phys. (in press)
} 